\title{
Reduced Order Modeling \& Controller Design for Mass Transfer in a Grain Storage System
}

\author{
Paramita Guha Sunita Mishra \\ Agrionics Division, Central Scientific Instruments Organisation, Chandigarh 160030, India
}

\begin{abstract}
This paper considers the problem of simulating the humidity distributions of a grain storage system. The distributions are described by partial differential equations (PDE). It is quite difficult to obtain the humidity profiles from the PDE model. Hence, a discretization method is applied to obtain an equivalent ordinary differential equation model. However, after applying the discretization technique, the cost of solving the system increases as the size increases to a few thousands. It may be noted that after discretization, the degree of freedom of the system remain the same while the order increases. The large dynamic model is reduced using a proper orthogonal decomposition based technique and an equivalent model but of much reduced size is obtained. A controller based on optimal control theory is designed to obtain an input such that the output humidity reaches a desired profile and also its stability is analyzed. Numerical results are presented to show the validity of the reduced model and possible further extensions are identified.
\end{abstract}

Keywords: Grain storage system, finite element method, modeling, reduced order modeling, proper orthogonal decomposition, optimal control, Lyapunov stability criteria.

\section{Introduction}

In recent years modeling, simulation and control of physical systems or processes have become crucial areas of active research. Increasing demands for reliable and detailed analysis of various practical problems has made numerical modeling and simulation of physical systems an important area of research. This is true for both analyses of the systems themselves through simulations, as well as for design of associated controllers for system or process control $^{[1]}$. Moreover, most practical physical systems are governed by partial differential equations (PDEs) and are of high-dimensional in nature. They appear in various application areas, such as thermal processes, chemical processes, agricultural \& biological systems, etc. ${ }^{[2,3]}$ These systems involve strong or weak interactions between different physical phenomena. Hence, lots of challenges are involved in modeling, simulation and controller design. In this work, modeling of a grain storage system is considered. Also, we have designed a controller to obtain an optimal input temperature such that the output humidity reaches to a desired profile.

The main objective of a good storage is to create suitable environmental conditions that provide protections to the grains or food products to maintain its quality and quantity. Health and quality of stored grain is mostly affected by attacks of insects, fungi, etc. The growth of insects can be controlled by controlling temperatures while controlled humidity can prevent the growth of mite, fungi. Hence, temperature as well as humidity plays very important role in storage systems ${ }^{[4]}$. Therefore, accurate predictions of them are quite necessary so that proper corrective actions can be taken.

Conducting experiments to study the moisture distributions in grain storage systems are expensive and time-

Regular paper

Manuscript received March 1, 2013; revised September 12, 2013 consuming. Hence, numerical simulations can be performed to predict them. There are several numerical tools available for this purpose like, finite difference (FD), finite element (FE), boundary element (BE) methods, etc. The most popular method is FE method because of its several advantage ${ }^{[5]}$. However, this procedure is also timeconsuming as the simulation time depends upon the number of nodes of the discretized model. To reduce the simulation time for this application, an equivalent model of reduced size can be developed which has almost same dynamic properties as the original one. Consequently model order reduction (MOR) is presently a very active area of recent research ${ }^{[6]}$, for both linear as well nonlinear systems. The area of MOR for practical distributed systems has been extensively developed. Several reduction techniques are available for them like, singular value decomposition $(\mathrm{SVD})^{[7]}$, balanced truncation, Krylov subspace projection ${ }^{[8-11]}$, proper orthogonal decomposition (POD) approach $^{[12-15]}$, etc.

In this work, dynamic equations for moisture of a grain storage system are simulated and an equivalent model with lesser number of variables is obtained. The approach involved discretization of governing equations with FE method and obtaining a reduced nonlinear dynamic system of much smaller size with almost same dynamic property as the original using POD method. A controller based on optimal control theory ${ }^{[16]}$ is designed for the reduced model so that the output reaches to a desired profile. It has also been shown that the controller acts fairly good for the original model as well. The paper is organized in the following way. In Section 2, the geometrical characteristics of the storage system are discussed in detail. FE modeling of the system is given in Section 3 and a reduced order model is developed in Section 4. A controller is designed in Section 5 and its stability is analyzed using Lyapunov stability criteria ${ }^{[17]}$. In Section 6 , the numerical results are 
given and finally the paper is concluded with Section 7 .

Table 1 Notations

\begin{tabular}{|c|c|c|}
\hline Parameters & Description & Unit \\
\hline$[C],[K]$ & Coefficient matrices & \\
\hline$D$ & Moisture diffusion coefficient & $\left(\mathrm{m}^{2} / \mathrm{s}\right)$ \\
\hline$E$ & Modulus of elasticity & $\left(\mathrm{N} / \mathrm{m}^{2}\right)$ \\
\hline$E_{b}$ & Activated energy & $(\mathrm{kJ} / \mathrm{mol})$ \\
\hline $\bar{F}$ & Input vector & \\
\hline$F_{1}$ & Positive definite matrix & \\
\hline$k$ & Permeability of the medium & $\left(\mathrm{m}^{2}\right)$ \\
\hline$k_{m} *$ & Coefficient of diffusion & \\
\hline$M^{*}$ & Activated moisture & $\left(\mathrm{kg}_{\text {water }} / \mathrm{kg}_{\text {grain }}\right)$ \\
\hline$\mu_{w}$ & $\begin{array}{c}\text { Dynamic viscosity of the } \\
\text { moisture }\end{array}$ & $\left(\mathrm{m}^{2}\right)$ \\
\hline$\nabla P$ & Pressure gradient & $(\mathrm{Pa} / \mathrm{m})$ \\
\hline$Q$ & Positive semidefinite matrix & \\
\hline$R$ & Gas constant & $(\mathrm{J} /(\operatorname{molK}))$ \\
\hline$r$ & Dimension of reduced model & \\
\hline$\sigma$ & Singular values & \\
\hline$S$ & Snapshot matrix & \\
\hline$U$ & Moisture concentration & $\left(\mathrm{kg}_{\text {water }} / \mathrm{kg}_{\text {grain }}\right)$ \\
\hline $\bar{U}_{0}$ & Initial humidity & $\left(\mathrm{kg} / \mathrm{m}^{3}\right)$ \\
\hline $\bar{U}^{*}$ & Desired humidity & $\left(\mathrm{kg} / \mathrm{m}^{3}\right)$ \\
\hline$U_{e q}(T)$ & $\begin{array}{l}\text { Equilibrium water } \\
\text { holding capacity }\end{array}$ & $\left(\mathrm{kg}_{\text {water }} / \mathrm{kg}_{\mathrm{dry} \text { _air }}\right)$ \\
\hline$u_{1}$ & Input function & $\mathrm{K}$ \\
\hline$u_{1}^{*}$ & Optimal input function & $\mathrm{K}$ \\
\hline$u_{w}$ & Water velocity & $(\mathrm{m} / \mathrm{s})$ \\
\hline$t$ & Time & $\mathrm{d}$ \\
\hline$t_{0}$ & Initial time & d \\
\hline$t_{f}$ & Final time & d \\
\hline$z_{0}$ & Initial reduced states & \\
\hline$z^{*}$ & Desired reduced humidity & \\
\hline
\end{tabular}

\section{Geometry and problem description}

For the present work, we have considered a three dimensional grain storage system as shown in Fig. 1, similar to that considered in [18]. The axisymmetrical view can be obtained if the system is cut along $z$-axis. The total capacity of the system is about 1.25 tones and its dimensions are given in Fig. 1. The storage system is made of galvanized iron. The simulations are performed for environmental conditions of Konkan region where humidity levels are quite high. Special care should be taken while controlling the moisture levels. Hence, proper predictions of them are very important.

\section{$3 \quad$ Finite element modeling}

The governing equation for moisture transport ${ }^{[19]}$ within the storage system is given by

$$
\frac{\partial U}{\partial t}+\nabla\left(U u_{w}\right)=\nabla D \nabla U
$$

The velocity $u_{w}$ can be written as

$$
u_{w}=-\frac{k}{\mu_{w}} \nabla P
$$

The pressure can be rewritten as ${ }^{[19]}$

$$
P=E\left(U-U_{e q}(T)\right) .
$$

The function $U_{e q}(T)$ can be taken as

$$
U_{e q}(T) \propto T .
$$

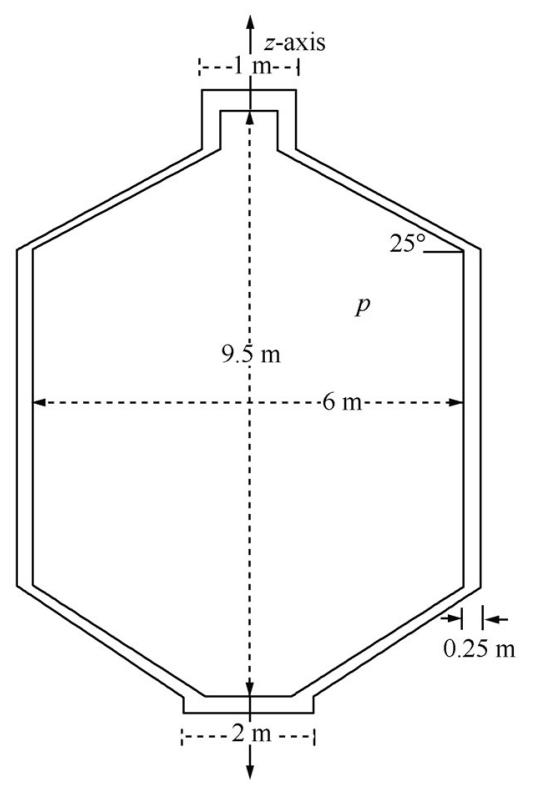

Fig. 1 Three dimensional problem domain

The proportionality constant can be obtained using [20]. Hence, from (1)-(3), we can write

$$
\begin{aligned}
\frac{\partial U}{\partial t}= & \frac{k E}{\mu_{w}} \nabla\left(U \nabla\left(U-U_{e q}(T)\right)\right)+\nabla D \nabla U= \\
& \frac{k E}{\mu_{w}} \nabla^{2} U+\nabla D \nabla U .
\end{aligned}
$$

The gradient of moisture diffusion coefficient can be written as ${ }^{[21]}$

$$
\nabla D=-k_{m^{*}} \frac{\mathrm{d} M^{*}}{\mathrm{~d} x} .
$$

The activated moisture content $M^{*}$ can be calculated from Boltzmann distribution as

$$
M^{*}=U \exp \left(-\frac{E_{b}}{R T}\right) .
$$

Here $E_{b}$ is calculated as

$$
E_{b}=35800-290 U \text {. }
$$

Further,

$$
\begin{aligned}
\frac{\mathrm{d} M^{*}}{\mathrm{~d} x} \approx & \left.\frac{\partial M^{*}}{\partial U}\right|_{T=\text { const }} \frac{\mathrm{d} U}{\mathrm{~d} x}= \\
& \left(\frac{R T+290 U}{R T}\right) \exp \left(-\frac{E_{b}}{R T}\right) .
\end{aligned}
$$

Now, combining (5) and (9)

$\frac{\partial U}{\partial t}=\left(\frac{k E}{\mu_{w}}-k_{m^{*}}\left(\frac{R T+290 U}{R T}\right) \exp \left(-\frac{E_{b}}{R T}\right)\right) \nabla^{2} U$. 
After applying FE method, a simplified model can be obtained as ${ }^{[5]}$

$$
[C] \frac{\partial \bar{U}}{\partial t}+[K] \bar{U}=\bar{F} u_{1}
$$

where $u_{1}$ is an input function dependent on temperature $(T)$.

\section{Model order reduction}

After obtaining the time-simulation of the system, a "snapshot matrix" $S$ is formed. Response states of the simulated data are collected as columns of $S$. An orthogonal basis for this matrix is obtained through singular value decomposition (SVD), given as

$$
V_{1}^{\prime} S V_{2}=\Sigma
$$

where $V_{1}^{m \times m}$ is an orthogonal matrix whose columns constitute an orthonormal "output" basis vector of the matrix $S^{m \times n}$. Similarly, columns of orthogonal $V_{2}^{n \times n}$ matrix form orthonormal "input" basis vector of $S$ and diagonal matrix gives the singular values arranged in decreasing order. If the first $r$ singular values $\sigma_{1}, \sigma_{2}, \cdots, \sigma_{r}$ are considered significant, then the first $r$ columns of $V_{1}$ taken as $V_{r}=\left[v_{1}, v_{2}, \cdots, v_{r}\right]$ form an orthogonal basis of the range space of the snapshot matrix $S$. As expected, the dimension $r$ of this subspace is found to be much smaller than the number of state variables. Projecting the overall dynamics on this subspace, given by ${ }^{[22]}$

$$
\bar{X}=V_{r} \bar{z}_{r}
$$

leads to a much smaller nonlinear model that captures most of the significant dynamics. The reduced model is given by

$$
\left[C_{r}\right] \frac{\partial \bar{z}}{\partial t}+\left[K_{r}\right] \bar{z}=\bar{F}_{r} u_{1}
$$

where $C_{r}=V_{r}^{\prime} C V_{r}, K_{r}=V_{r}^{\prime} K V_{r}$ and $\bar{F}_{r}=V_{r}^{\prime} \bar{F}$.

\section{The control problem}

Here we consider a control problem which transfers the initial humidity $\bar{U}_{0}$ to a desired humidity profile represented by a nodal vector $\bar{U}^{*}$. As the vector $\bar{U}_{0}$ and $\bar{U}^{*}$ lie in the span of projection matrix $V_{r}$, the corresponding reduced states $z_{0}$ and $z^{*}$ can be derived using respectively.

$$
\begin{aligned}
& \bar{U}_{0}=V_{r} z_{0} \\
& \bar{U}^{*}=V_{r} z^{*} .
\end{aligned}
$$

In case, the vectors $\bar{U}_{0}$ and $\bar{U}^{*}$ do not lie in the span of $\bar{V}_{r}$, then $z_{0}$ and $z^{*}$ will imply their respective least square approximations in the projected subspace.

\subsection{Controller design}

An optimal control based performance index can be taken as $^{[23-25]}$

$$
J=\frac{1}{2} e^{\prime}\left(t_{f}\right) F_{1}\left(t_{f}\right) e\left(t_{f}\right)+\frac{1}{2} \int_{t_{0}}^{t_{f}}\left[z^{\prime}(t) Q(t) z(t)+u_{1}^{2}(t)\right] \mathrm{d} t .
$$

Here error between the actual and desired states is given as

$$
e(t)=z(t)-z^{*}
$$

The optimal control input can be obtained as

$$
u_{1}^{*}=-\bar{F}_{r}^{\prime} \hat{P} z^{*}
$$

where $\hat{P}$ is an $n \times n$ symmetric positive definite matrix with $t \in\left[t_{0}, t_{f}\right]$, given as

$$
\hat{\dot{P}}(t)=-\hat{P}(t) K_{r}-K_{r}^{\prime} \hat{P}(t)-Q(t)+\hat{P}(t) \bar{F}_{r} \bar{F}_{r} \hat{P}(t) .
$$

The above equation is solved using backward difference method with $\hat{P}(t \rightarrow \infty)=0$.

\subsection{Stability analysis}

A Lyapunov function in terms of error $e(t)$ can be chosen as

$$
\Phi(t)=\frac{1}{2} e^{\prime}(t) e(t)
$$

which is positive definite. For the stability analysis, the first order derivative of $\Phi(t)$ is calculated.

$$
\dot{\Phi}(t)=e^{\prime}(t) \dot{e}(t)=e^{\prime}(t) \dot{z}(t) .
$$

Now, using (18), (19) and (22)

$$
\begin{aligned}
\dot{\Phi}(t)= & {\left[z^{\prime}(t)-z^{* *}\right]\left[-K_{r} z(t)+\bar{F}_{r} u^{*}\right]=} \\
& -z^{\prime}(t) K_{r} z(t)+z^{\prime *} K_{r} z(t)+z^{*} \bar{F}_{r} u_{1}^{*}(t)- \\
& z^{\prime *} \bar{F}_{r} u_{1}^{*}(t)= \\
& -\left(z^{\prime}(t)-z^{*}\right) K_{r} z(t)= \\
& -e^{\prime}(t) K_{r}\left(e(t)+z^{*}\right)= \\
& -e^{\prime}(t) K_{r} e(t)-e^{\prime}(t) K_{r} z^{*} \leqslant 0 .
\end{aligned}
$$

\section{Numerical results and discussion}

The proposed methodology is applied to a twodimensional axisymmetric geometry. The geometry can be obtained by taking an intersection of Fig. 1 along $z$-axis. The domain is discretized using triangular FE mesh with 7050 nodes excluding the boundary. Here the number of elements is 15008 . The meshed domain is shown in Fig. 2. The size of the ordinary differential equation (ODE) model is same as the number of nodes inside the geometry. Hence, size of the ODE model will be 7050 . Standard values of constants are taken and ambient conditions are taken for the simulation purpose. Rice is kept inside the storage system for the analysis purpose. The simulation time is taken from 1st March to 4th April for Konkan region, Maharashtra, India. The initial value of moisture is taken as $60 \%$ at $25^{\circ} \mathrm{C}$. Zero boundary conditions are applied here. However, it can be verified that this methodology can be applied for any other boundary conditions.

To generate the reduced model, the original model (12) is simulated with an impulse input. Here, in order to capture the widest frequency characteristics, the above input condition is taken. For simulations, backward difference is used, with 40 time steps are chosen for the computation 
and each step is taken as $1 \mathrm{~d}$. At each time-step, matrix $K$ is dependent on humidity and the resulting nonlinear algebraic equation system is solved using the Newton-Raphson method. Finally, those 40 humidity profiles for the timesteps or humidity snapshots are stored as columns of matrix $S$. Rank of the matrix $S$ is found as 18, beyond which the magnitudes of the singular values are negligible. This leads to the reduction of original nonlinear model to another model of (14) but with a much reduced size of 18. As a validation, the reduced model of the form (14) was again simulated with an impulse input $\delta(t)$ and the nodal humidity profiles were recomputed using (13). The humidity profiles were found to agree satisfactorily with those computed originally from the unreduced model given by (12). It has been observed that at $t=40 \mathrm{~d}$, the error is around $1.15 \%$. The errors are due to the neglected higher order dynamics. The humidity profiles for both original and reduced models at an arbitrary point $P_{1}$ are given in Fig. 3 . The humidity profiles are obtained with an initial value of relative humidity is $60 \%$ and temperature is kept at $25^{\circ} \mathrm{C}$.

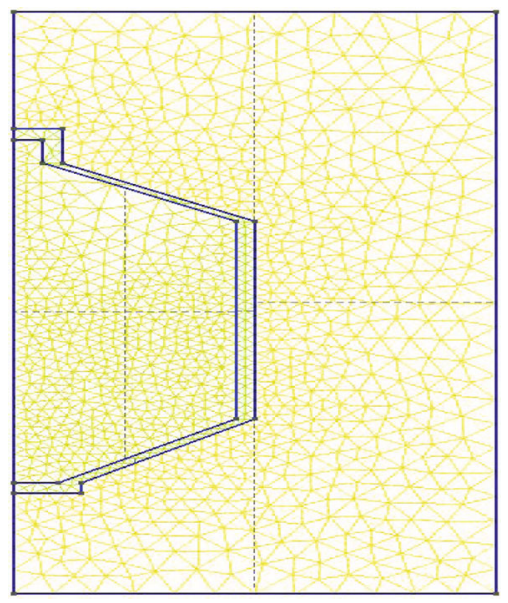

Fig. 2 Meshed domain

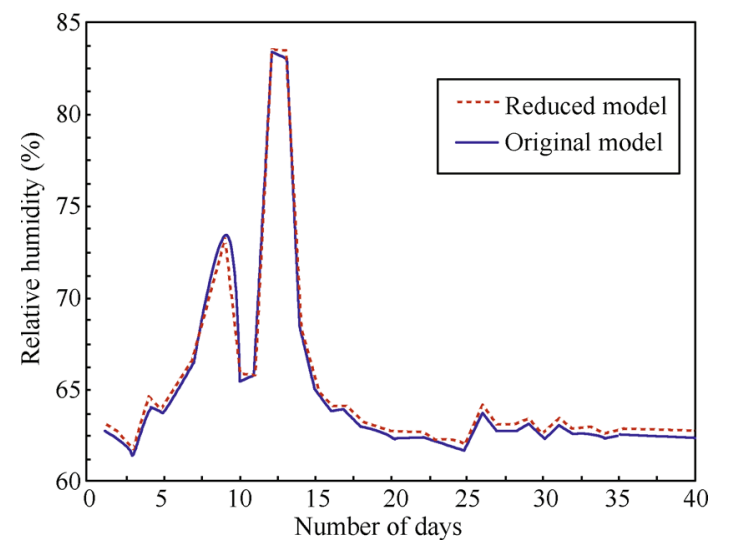

Fig. 3 Humidity profiles at $P_{1}$ point

The time taken to simulate the original model for $40 \mathrm{~s}$ is around $4.30 \mathrm{~min}$, while the reduced model takes $50 \mathrm{~s}$. The simulations are performed on $1 \mathrm{~GB}, 1.85 \mathrm{GHz}$, core- 2 duo machines. Absolute time and their comparisons would be clear if the entire implementation is done in $\mathrm{C} /$ Fortan. For the purpose of simulation of the process Scilab ${ }^{[26]}$ software implementation is used. For the discretization purpose, $\mathrm{Gmsh}^{[27]}$ software is used. A comparison between the proposed and conventional methods can be done in terms of the size of the problem presented to any computer and the computational effort or complexity of the solution processirrespective of the hardware. In the present case, the reduction in computational effort was from execution of nonlinear simulations with 7050 dynamic variables to execution of almost similar simulations with 18 variables.

Now, the reduced model (14) is simulated along with the optimal control law (19) which transfers an arbitrarily chosen $z_{0}$ to a desired target $z^{*}$. Here all the components of $z^{*}$ are taken as 1000 . The desired humidity profiles $U^{*}$ corresponding to $z^{*}$ can be obtained using (16). The nodal humidity profiles can be obtained from $z(t)$. Here, the Riccati equation (20) is solved using Secant method and the magnitude of the temperature is obtained as $26.32^{\circ} \mathrm{C}$ at $t=40 \mathrm{~d}$, being a representative time. The output profiles of the original and reduced model, when represented in full coordinates, at $t=40 \mathrm{~d}$, after applying the optimal control law match almost perfectly. Those are shown in Fig. 4. It has been observed that the error between the outputs of both models, when represented in full coordinates at different time instants is around $1.856 \%$. Hence, we can conclude that the controller acts satisfactorily.

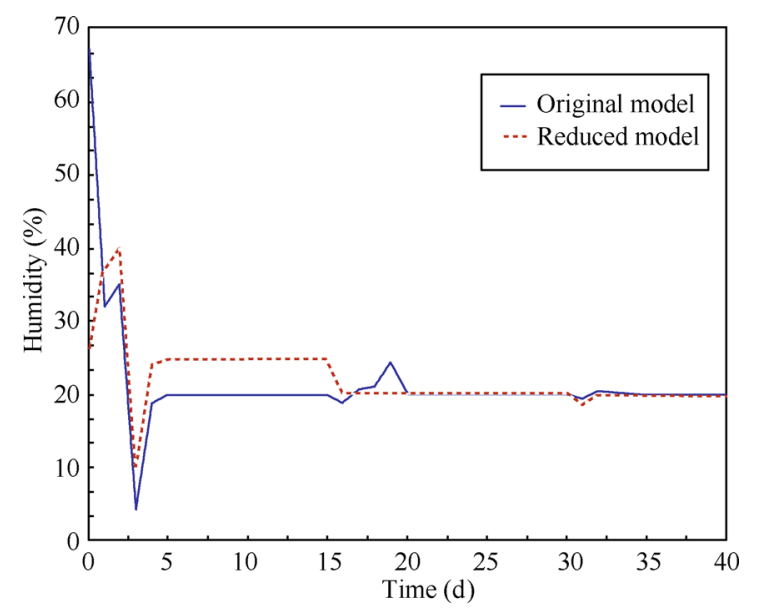

Fig. 4 Humidity profile with controller

\section{Conclusions}

In this work, a modeling strategy for a grain storage system is presented. Here humidity is taken as variable to be modeled. FEM is used for discretization purpose thus making the approach suitable to more complicated geometries. A reduced order model is obtained using proper orthogonal decomposition technique. It has been observed that the reduced model has almost same dynamic properties as the original one. A controller based on optimal control theory is developed and its stability is analyzed using Lyapunov stability criteria. It can be observed that the moisture level was quite high in the actual system, which is a favorable condition for infestation. However, using the controller it is controlled to a certain level. Although the controller is designed for the reduced model, it works satisfactorily for the original system as well. This is the main contribution of this 
work. This work can be extended to obtain an equivalent reduced model where dynamic equations involve coupled temperature and moisture.

\section{References}

[1] B. N. Datta. Numerical Methods for Linear Control Systems: Design and Analysis, New York: Elsevier Academic Press, 2003.

[2] A. W. Leung. Systems of Nonlinear Partial Differential Equations: Applications to Biology and Engineering, New York: Springer, 1989.

[3] T. Roubíček. Nonlinear Partial Differential Equations with Applications, New York: Springer, 2005.

[4] D. W. Sun, J. L. Woods. Low temperature moisture transfer characteristics of wheat in thin layers. Transactions of the American Society of Agricultural Engineers, vol. 37, no. 6, pp. 1919-1926, 1994.

[5] J. N. Reddy. An Introduction to the Finite Element Method, New York, USA: McGraw Hill, Inc., 1993.

[6] T. T. Lee, F. Y. Wang, R. B. Newell. Robust model-order reduction of complex biological processes. Journal of Process Control, vol. 12, no. 7, pp. 807-821, 2002.

[7] Z. Li, X. Lu, W. P. Shi. Process variation dimension reduction based on SVD. In Proceedings of the 2003 International Symposium on Circuits and Systems, IEEE, Bangkok, vol. 4, pp. IV-672-IV-675, 2003.

[8] R. W. Freund. Krylov-subspace methods for reduced-order modeling in circuit simulation. Journal for Computational and Applied Mathematics, vol.123, no.1-2, pp.395-421, 2000.

[9] B. N. Datta. Krylov subspace methods for large-scale matrix problems in control. Future Generation Computer Systems, vol. 19, no. 7, pp. 1253-1263, 2003.

[10] E. J. Grimme. Krylov Projection Methods for Model Reduction, Ph. D. dissertation, University of Illinios at Urbana Champaign, Urbana, IL, USA, 1997.

[11] S. Gugercin. An iterative SVD-Krylov based method for model reduction of large-scale dynamical systems. In Proceedings of the 44th IEEE Conference on Decision and Control, and the European Control Conference, IEEE, Spain, pp. 5905-5910, 2005.

[12] A. Chatterjee. An introduction to the proper orthogonal decomposition. Special Section: Computational Science, Current Science, vol. 78, no. 7, pp. 808-817, 2000.

[13] S. Y. Shvartsman, C. Theodoropoulos, R. Rico-Martínez, I. G. Kevrekidis, E. S. Titi, T. J. Mountziaris. Order reduction for nonlinear dynamic models of distributed reacting systems. Journal of Process Control, vol.10, no. 2-3, pp. 177-184, 2000.

[14] C. W. Rowley. Model reduction for fluids, using balanced proper orthogonal decomposition. International Journal of Bifurcation and Chaos, vol. 15, no. 3, pp. 997-1013, 2005.

[15] Y. Zhai, L. Vu-Quoc. Analysis of power magnetic components with nonlinear static hysteresis: Proper orthogonal decomposition and model reduction. IEEE Transactions on Magnetics, vol. 43, no. 5, pp. 1888-1897, 2007.

[16] Y. Tenne. An optimization algorithm employing multiple metamodels and optimizers. International Journal of $\mathrm{Au}$ tomation and Computing, vol. 10, no. 3, pp. 227-241, 2013.

[17] E. M. Jafarov. On stability delay bounds of simple inputdelayed linear and non-linear systems: Computational results. International Journal of Automation and Computing, vol. 10, no. 4, pp. 327-334, 2013.

[18] N. J. Thakor, S. C. Patil, A. A. Sawant, S. D. Sawant. Influence of storage period on physical properties and quality of paddy stored in GIC silo and bag storage under humid Konkan climatic conditions. Journal of Agricultural Engineering, vol. 49, no. 1, pp. 43-47, 2012.
[19] J. Zhang. Modelling and multi-objective optimal control of batch processes using recurrent neuro-fuzzy networks. International Journal of Automation and Computing, vol. 3, no. 1, pp. 1-7, 2006.

[20] P. Chakraborty, S. Banerjee. Optimization of extrusion process for production of expanded product from green gram and rice by response surface methodology. Journal of Scientific and Industrial Research, vol.68, no. 2, pp. 140-148, 2009 .

[21] J. C. Alvarez. Evaluation of Moisture Diffusion Theories in Porous Materials, Master dissertation, Virginia Polytechnic Institute and State University, USA, 1998.

[22] P. Guha, M. un Nabi. Model reduction and controller design for a nonlinear heat conduction problem using finite element method. International Journal of Automation and Computing, vol. 9, no. 5, pp. 474-479, 2012.

[23] M. un Nabi, P. Guha. A control law for a nonlinear heat conduction problem on nontrivial domains using FEM. In Proceedings of the 17th Mediterranean Conference on Control and Automation, IEEE, Thessaloniki, pp. 320-323, 2009.

[24] D. S. Naidu. Optimal Control Systems, London, UK: CRC Press, 2002.

[25] P. Guha, M. un Nabi. Optimal control of a nonlinear induction heating system using a proper orthogonal decomposition based reduced order model. Journal of Process Control, vol. 22, no. 9, pp. 1681-1687, 2012.

[26] http://www.scilab.org.

[27] http://www.geuz.org/gmsh/.

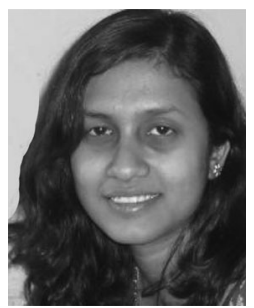

Paramita Guha graduated from Jalpaiguri Goverment Engineering College, India in 2001. She received her master degree from Bengal Engineering College (Deemed University), India in 2003 and Ph. D. degree from Indian Institute of Technology (IIT), Delhi, India in 2013, both in electrical engineering. She is presently working as a scientist in CSIR-Central Scientific Instruments Organisation, Chandigarh, India. She has published several papers in international journal and conferences.

Her research interests include distributed parameter systems, coupled systems, modeling and simulation, model reduction and control theory.

E-mail: paramguha@gmail.com (Corresponding author)

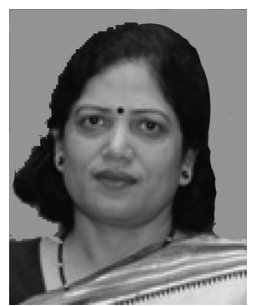

Sunita Mishra did her graduation and post-graduation in physics in the year 1983 and 1985, respectively from Allahabad University, Allahabad. She obtained her $\mathrm{Ph}$. D. degree in physics-electronics engineering from Institute of Technology, Banaras Hindu University, Varanasi in 1993 At present she is working as a principal scientist in CSIR-Central Scientific Instruments Organisation, Chandigarh. She has completed projects in the area of agricultural electronics, gas sensors, semiconductor devices and NIR spectroscopy. She has published about 25 papers in journals and conference proceedings. She is life member of semiconductor society of India, Solar Energy Society of India and Material Research Society of India and the fellow of the Institution of Electronics and Telecommunication Engineers (IETE).

Her research interests include sensors, semiconductor devices, and NIR spectroscopy.

E-mail: sunita_mishra@csio.res.in 